\title{
Design of Electrocardiograph Signal Simulator
}

\author{
Catur Suharinto ${ }^{\#}$, Anwar Budianto, Nugroho Tri Sanyoto \\ IPS Medik RSUP Dr. M. Djamil Padang \\ Jl. Perintis Kemerdekaan, Padang, Indonesia \\ STTN-BATAN \\ Jl. Babarsari, Caturtunggal, Kec. Depok, Kabupaten Sleman, Daerah Istimewa Yogyakarta, Indonesia \\ \#catur3414@gmail.com, taufad@yahoo.com, trisanyotonugroho@yahoo.co.id,
}

\begin{tabular}{l}
\hline \multicolumn{1}{c}{ Article Info } \\
\hline Article History: \\
Received June 9, 2019 \\
Revised July 20, 2019 \\
Accepted Jan 11, 2020
\end{tabular}

Keywords:

ECG Simulator

Microcontroller

Calibrator

Phantom

DAC

\begin{abstract}
Medical equipment functional test and calibration is a routine activity that must be carried on periodically. Electrocardiograph (ECG) requires an ECG phantom to calibrate the function. This calibrator is commonly called ECG signal simulator. The purpose of this study is to design a simple ECG signal simulator with ten leads of signals that can be used to test ECG recorders with standard recording procedures. With the ECG signal simulator that was designed and made, the development of signal patterns can be made as needed. The normal human cardiac signal displayed on the ECGSIM software. The potential value that displayed on ECGSIM software can be extracted manually and assembled as a flash program of microcontroller, so this microcontroller will generate some digital code by each parallel port. This digital code then converted as an analog signal by DAC. The electrocardiograph signal simulator output is an analog signal that identical with each lead according to the recording method of bipolar, unipolar and precordial of ECG. This analog signal was tested using a standard ECG recorder. It is proved that the simulator is able to generate an electrical signal in accordance with the characteristics of the human cardiac signal displayed on ECGSIM software. The results of human electrocardiograph signal simulator design are a device that generates electrical signals with output specifications that correspond to the bioelectric signals of the human heart. The statistical test showed that the p-value is more than 0.05 . It is mean that there is no significant difference between the design and standard. The signals pattern has met the specification of ECGSIM signal.
\end{abstract}

\author{
Corresponding Author: \\ Catur Suharinto \\ IPS Medik RSUP Dr. M. Djamil Padang \\ Jl. Perintis Kemerdekaan, Padang, Indonesia \\ Email: catur3414@gmail.com
}

This work is an open-access article and licensed under a Creative Commons Attribution ShareAlike 4.0 International ( $\underline{\mathrm{CC} B Y-S A}$ 4.0 $)$.

\section{INTRODUCTION}

Every human's heart has difference cardiac signal each other. Amplitude, BPM and signal pattern are the difference. This difference causes difficulties to get a standard ECG signal, in order to study or calibrate the ECG recorder. Electrocardiograph Signal Simulator is an equipment that generates some analog signal that has a similar specification and consistent pattern. Nowadays, human cardiac simulator is very expensive, because it's made by the foreign medical equipment industry. Besides expensive, the dependence of the simulator's spare parts also occurs. Any malfunction on this simulator will take a long time to repair. Because of very important, it needs to build a low-cost human Electrocardiograph Signal Simulator to meet the needs of the hospital and health faculty. This human cardiac simulator can generate some analog signal that looks like a cardiac signal that appears on the ECGSIM software but it may set the BPM 30 to 120 and choose an irregular signal on it.

Sangita et al. [1] have developed an ECG simulator of a microcontroller-based hardware ECG simulator which generates real-time analog ECG signals in the range of $0-5$ volt. The synthetic ECG signal generated by the simulator can be used for testing and calibration of medical instruments, biomedical experiments and research in laboratories. This ECG signal simulator only produces one signal, but the shape of the signal will change according to the input code. Berbari.[2] said that the ECG machine has the ability to produce $12 \mathrm{ECG}$ recording patterns cardiac signal from 9 
measurement points on the surface of human skin. ECG signal is the result of the amplification of some cardiac bioelectric signals that scanned on the surface of the skin using a differential amplifier. ECG pattern is obtained by applying the three standard scanning methods there are bipolar, unipolar and precordial. There are nine measurement points on the surface of human skin, consist of the right arm, left arm, left leg and six-point on the surface of the human chest. Fig 1 shows the cardiac bioelectric recording method.



(a)

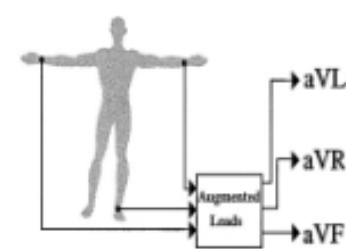

(b)

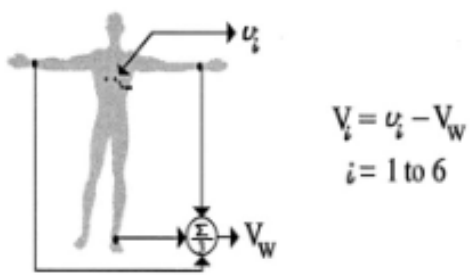

(c)

Fig.1. Recording method (a) Bipolar; (b) Unipolar; (c) Precordial.

Zhang. [3] reports a precise design and development for ECG signal generator for testing and calibration of the electrocardiogram equipment, ECG signal processing system, and heart teaching tool. It can create a guide two signal of time and profile features lead II signal across a range of ECG drawings of heart rate within 45 to 185 devices in the first device of steps. Valais et al. [4] have completed a practical ECG'S device that can be used to calibrate and test ECG recorders. Various beat-rates or ECG rhythms $(60,80,120,150$, and $180 \mathrm{bits} / \mathrm{min}$ ) of the normal ECG waveform produced by analog electronics the ECG 10 standard electrode recording was successful. In this study, four tapping signals were generated. Oosterom et al. [5] have developed ECGSIM software that can display human heart signal patterns. Using this tool, amplitude measurement for each signal becomes easier. R. Firmansyah et al. [6] have used ECG phantom to test Monitoring Heart Rate And Temperature Based On the Internet of Things. Simulators can also be used in research related to ECG, for example, Alvionita et al. [7] who has used ECG bioelectric signals.

\section{MATERIALS AND METHODS}

The first thing to do in designing this simulator is to prepare a vector array which is the amplitude of the heart signal at the standard ECG tapping point. ECGSIM is the answer to that problem. Using measurement tools, we can measure the amplitude of the cardiac signal on the ECGSIM monitor as Fig 2.

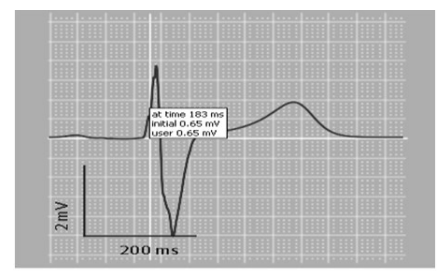

Fig 2. Amplitude Signal Measurement for ECGSIM Software.

Amplitude data that has been collected from the ECGSIM software is stored as a database as Fig 3 is assembled as a flash the program, the microcontroller can generate digital amplitude data on a parallel port.

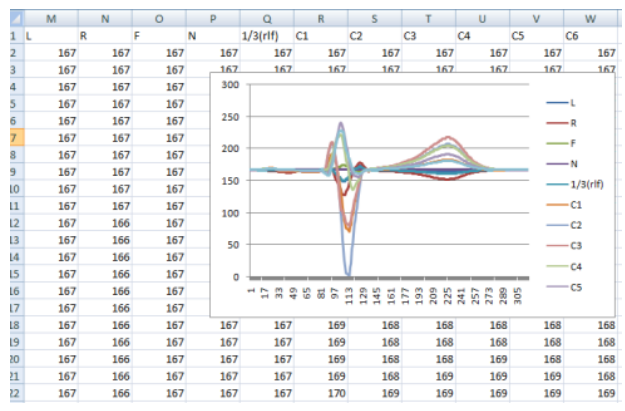

Fig.3. Amplitude data

\section{A. Hardware Design}

Every AT89S52 microcontroller has 4 parallel ports. Every port can be programmed as input/output (I/O) to generate digital condition on any 8 bits pin. ECG recording requires 9 measurement points on a standard recording method, requires 3 microcontrollers to generate 9 analog signals. To set the BPM, adding one more microcontroller as a user interface is necessary to connect it with push-button and LCD monitor as Fig 4.

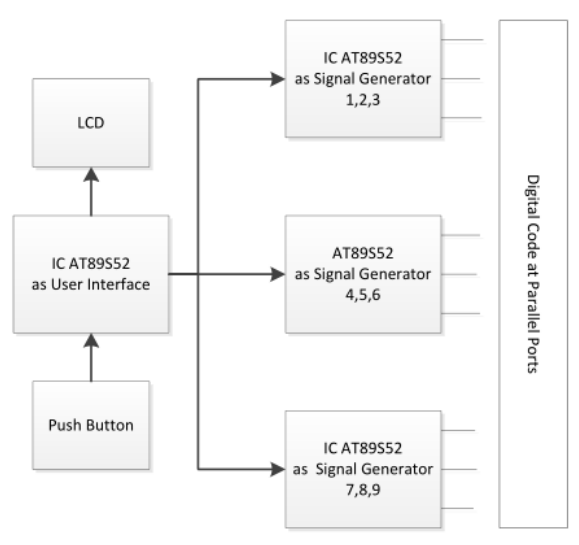

Fig 4. AT89S52 microcontroller Wiring Connecting of User Interface and Signal Generator 


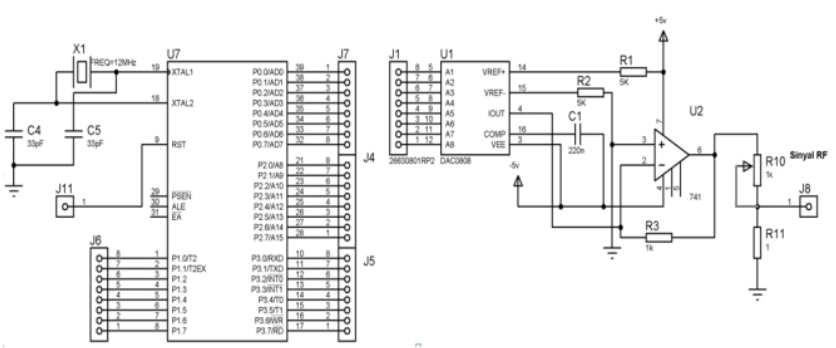

Fig 5. AT 89S52, DAC, I/V Converter and Attenuator circuit

The AT89S52 microcontroller converts the cardiac amplitude code in a flash program into a digital condition at parallel output. DAC converts the digital code generated by the microcontroller into an analog signal. Fig 5 shows the microcontroller circuit and DAC circuit. DAC 0808 is an 8-bit monolithic DAC. Nine DAC 0808 is needed to convert digital code from the parallel port of each AT89S52 microcontroller becomes an analog signal shown in Fig 5.



Fig 6. Block diagram of the ECG signal generator

AT89S52 IC's connected to DAC. Each 3 port of microcontroller will be connected to 3 DAC as shown in Fig 6. The electrical current output of DAC will be converted to an analog voltage by $\mathrm{I} / \mathrm{V}$ converter. The output of DAC can calculate by Eq.1 and Eq.2. Calculation of the electrical current (Iin) to electrical voltage (Vout) by LM741 IC's using feedback resistor R3 can be calculated by Eq.3.

$I o=K\left(\frac{A 1}{2}+\frac{A 2}{4}+\frac{A 3}{8}+\frac{A 4}{16}+\frac{A 5}{32}+\frac{A 6}{64}+\frac{A 7}{128}+\frac{A 7}{256}\right)$

$K=\left(\frac{\text { Vreff }}{2}\right)$

Vout $=\operatorname{Iin} . R 3$

The output of $\mathrm{I} / \mathrm{V}$ converter with $\mathrm{DAC}$ at the maximal condition of digital input (A1-A8) FF 8 is 4,98V. As shown on ECGSIM software, the maximal amplitude of the cardiac signal are $1,3 \mathrm{mV}$, this signal measured at V5. The minimal voltage of the cardiac signal show at $\mathrm{V} 2$ is $-3,2 \mathrm{mV}$. The range from the maximal and minimal signal is $4,5 \mathrm{mV}$. To meet the value, attenuator R10 and R11 must divide the maximal value of DAC output equal to $1 / 1106$.

\section{B. Software Design}

Fig 7a. show the microcontroller flowchart as the user interface. BPM values can be read on a 16x2 LCD monitor. The binary code is generated by port.0 AT89S52 IC as a user interface that provides information to other AT89S52 IC's like a signal generator to set the delay for signal generation indicating BPM values.

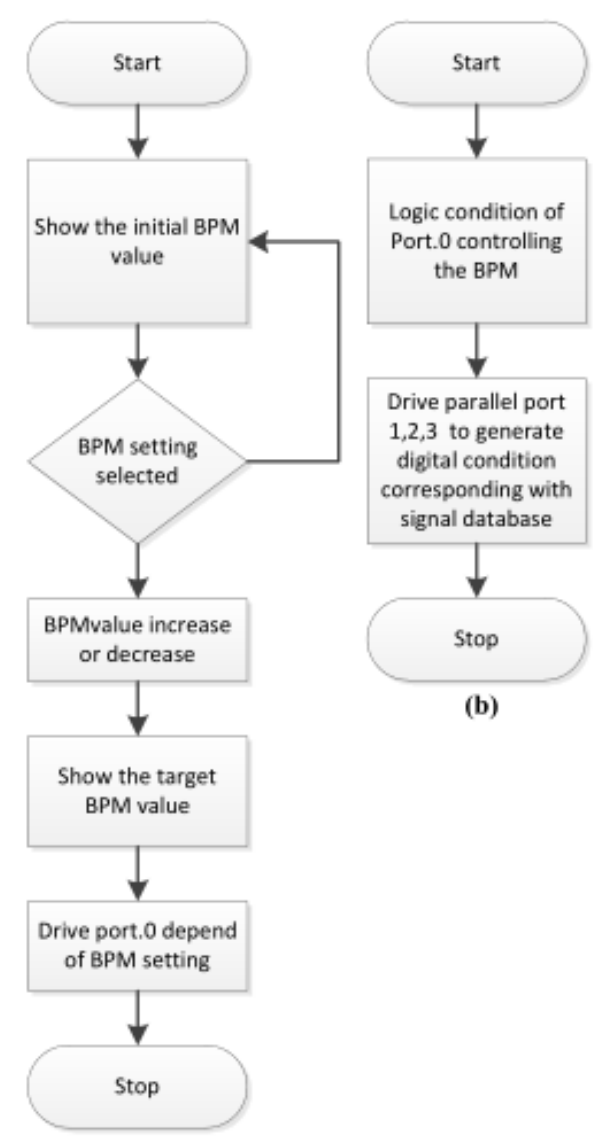

(a)

Fig 7. Flow charts; (a) Microcontroller program as user Interface; (b) Microcontroller program as signal generator.

Binary output from Microcontroller as a signal generator connected to port controlling BPM. Amplitude signal generated in accordance with the signal database on flash program. Fig 7b. representing the flowchart of microcontroller as a signal generator.

\section{RESULTS}

The results of human Electrocardiograph Signal Simulator design are a device that generates electrical signals with output specifications that correspond to the bioelectric signals of the human heart. Innomed TM 12 ECG coupling machine is used 
to test the output signal. Testing consists ECG pattern chosen signal output from the simulator signal shown in Fig 8.

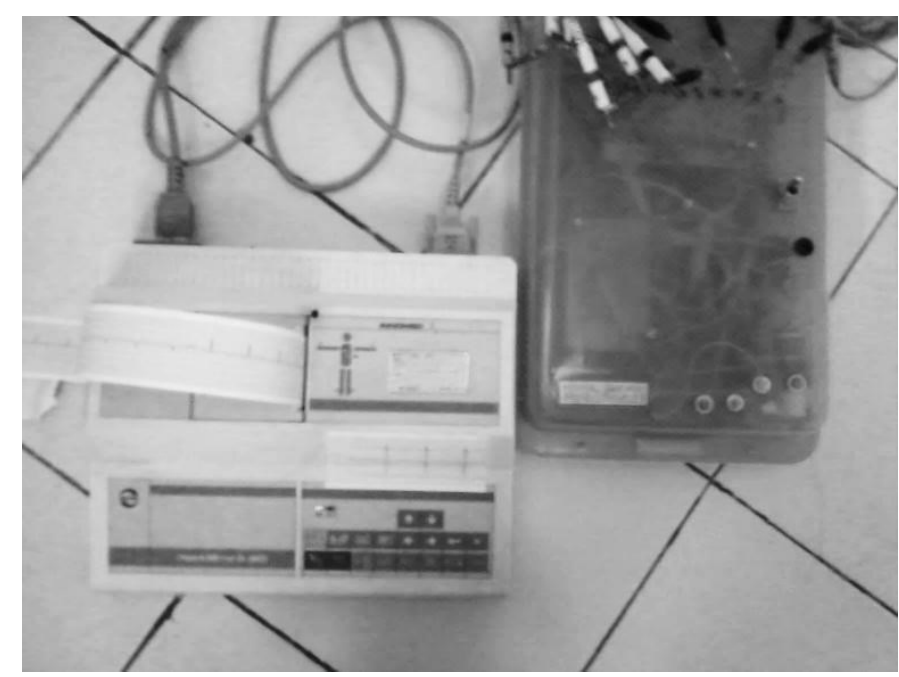

Fig 8. Testing Human Cardiac Simulator Using ECG Recorder

Testing the simulator output signal pattern according to the monopolar, bipolar and precordial standard ECG recording methods using ECG Recorder. Bipolar consists of lead I, lead II and lead III, unipolar there are aVR, aVL and aVF, precordial methods consist of V1, V2, V3, V4, V5 and V6. Fig 3 showing the recording result of the human cardiac simulator and compare it with ECGSIM standard signal pattern. Fig 9 shows the result of the human electrocardiograph signal simulator with irregular patterns.



Fig 9. Recording Irregular BPM Pattern Result of Human Cardiac Simulator

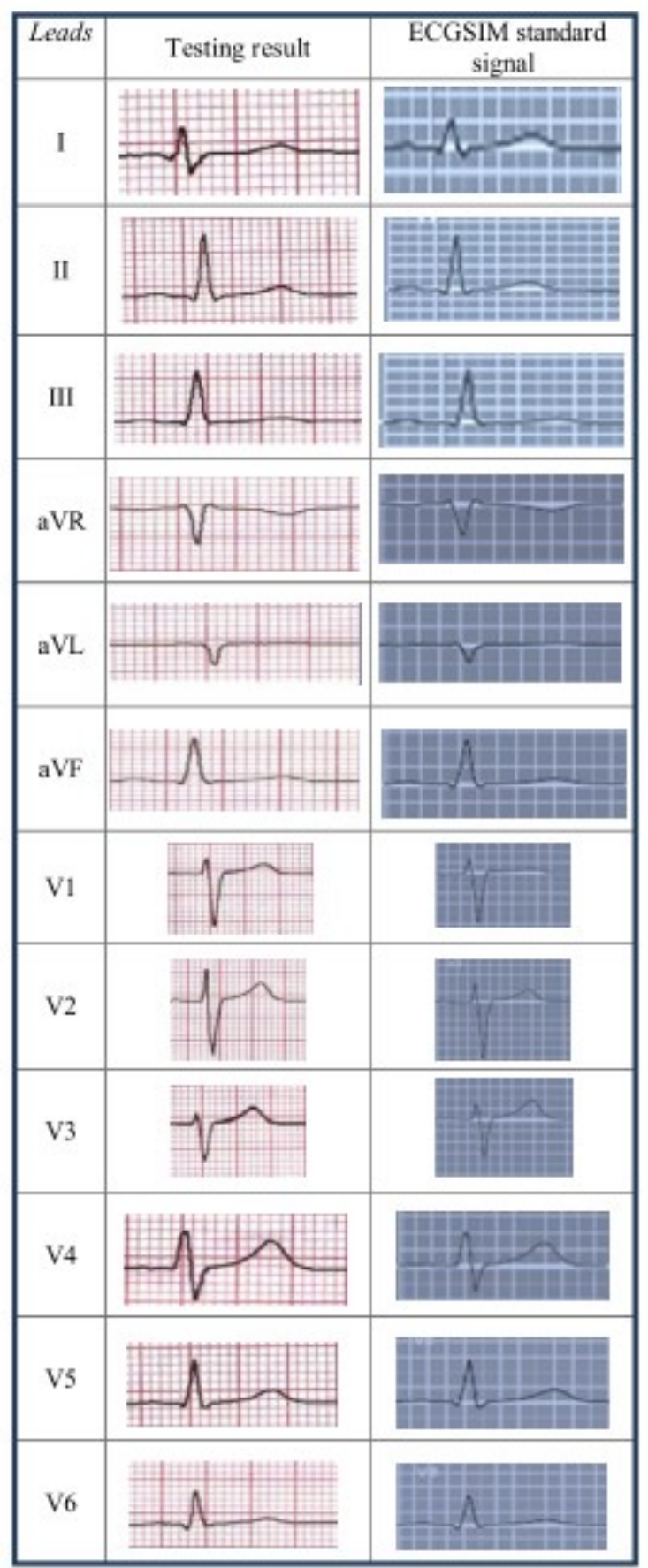

Fig 10. Signal Comparison

The purpose of this study is to create a simulator that can produce electrical signals similar to heart signals as displayed in the ECGSIM software. Shown in Fig 10 that the signal generated by the simulator has a similarity to the ECGSIM signal. For the purpose of validation, it is necessary to take measurements on each signal. Measurement results for each signal can be seen in Table 1 . To find out the similarity of the signal patterns generated between the simulator and the 
ECGSIM signal, a statistical analysis is needed. Table 2 shows the $\mathrm{t}$-Test for that data.

Table 1. Amplitude Measurement

\begin{tabular}{|c|c|c|c|c|c|}
\hline $\begin{array}{c}\text { ECG } \\
\text { Signal } \\
\end{array}$ & $\begin{array}{c}\text { Simulator } \\
(\mathrm{mV})\end{array}$ & $\begin{array}{c}\text { ECGSIM } \\
(\mathrm{mV})\end{array}$ & $\begin{array}{c}\text { ECG } \\
\text { Signal } \\
\end{array}$ & $\begin{array}{c}\text { Simulator } \\
(\mathrm{mV})\end{array}$ & $\begin{array}{c}\text { ECGSIM } \\
(\mathrm{mV})\end{array}$ \\
\hline \multirow{5}{*}{ I } & 0 & 0 & \multirow{5}{*}{ V1 } & 0 & 0 \\
\hline & -0.1 & -0.1 & & 0.5 & 0.5 \\
\hline & 0.4 & 0.4 & & -2 & -2 \\
\hline & -0.3 & -0.2 & & 0.1 & 0.1 \\
\hline & 0.2 & 0.2 & & -0.6 & -0.6 \\
\hline \multirow{5}{*}{ II } & 0.1 & 0.1 & \multirow{5}{*}{ V2 } & 0 & 1 \\
\hline & -0.1 & -0.1 & & 1.1 & 1 \\
\hline & 1.9 & 1.8 & & -2.6 & -2.7 \\
\hline & -0.1 & -0.1 & & 0.1 & 0.1 \\
\hline & 0.3 & 0.3 & & 0.6 & 0.6 \\
\hline \multirow{5}{*}{ III } & 0.1 & 0.1 & \multirow{5}{*}{ V3 } & 0 & 0 \\
\hline & 0.1 & 0.1 & & 0.5 & 0.5 \\
\hline & 1.5 & 1.5 & & -1.4 & -1.4 \\
\hline & 0 & 0 & & 0.1 & 0.1 \\
\hline & 0.1 & 0.1 & & 0.7 & 0.7 \\
\hline \multirow{5}{*}{ VR } & -0.1 & -0.1 & \multirow{5}{*}{ V4 } & 0 & 0 \\
\hline & 0.1 & 0.1 & & 0 & 0 \\
\hline & -0.7 & -0.6 & & -0.8 & -0.8 \\
\hline & 0.1 & 0.1 & & -0.7 & -0.7 \\
\hline & -0.1 & 0.1 & & 0.6 & 0.6 \\
\hline \multirow{5}{*}{ AVL } & 0 & 0 & \multirow{5}{*}{ V5 } & 0.1 & 0.1 \\
\hline & 0 & 0 & & -0.1 & -0.1 \\
\hline & -0.4 & -0.4 & & 1 & 1 \\
\hline & 0 & 0 & & -0.1 & -0.1 \\
\hline & 0 & 0 & & 0.3 & 0.3 \\
\hline \multirow{5}{*}{ VF } & 0 & 0 & \multirow{5}{*}{ V6 } & 0.1 & 0.1 \\
\hline & 0.1 & 0.1 & & -0.1 & -0.1 \\
\hline & 1.2 & 1.1 & & 1 & 1 \\
\hline & 0 & 0 & & -0.1 & -0.1 \\
\hline & 0.1 & 0.1 & & 0.2 & 0.2 \\
\hline
\end{tabular}

Table 2. t-Test result

\begin{tabular}{lrr}
\hline \multicolumn{1}{c}{ Parameter } & Simulator & ECGSIM \\
\hline Mean & 0.048333333 & 0.065 \\
Variance & 0.468980226 & 0.476211864 \\
Observations & 60 & 60 \\
Pooled Variance & 0.472596045 & \\
Hypothesized Mean & 118 & \\
Difference & & \\
Df & -0.132789683 & \\
t Stat & 0.447292947 & \\
P(T<=t) one-tail & 1.657869523 & \\
t Critical one-tail & 0.894585893 & \\
P $(\mathrm{T}<=\mathrm{t})$ two-tail & 1.980272226 & \\
t Critical two-tail & & \\
\hline
\end{tabular}

\section{DISCUSSION}

The human cardiac signal simulator has been made and tested using an ECG recording machine. In table 2 it appears that $\mathrm{t}$ Stat is smaller than $\mathrm{t}$ Critical two-tail, with a value $0.133<1.980$. This value indicates that the two sets of signals have similarities.

The results of this study can be compared with previous researchers. Zhang [3] makes a simulator circuit that can produce one signal. Oosterom et al. [5] have created software that can display ECG signals in full on the monitor screen. This research has produced a simulator circuit that can generate cardiac signals whose values are similar to the cardiac signals displayed on ECGSIM.

\section{Conclusion}

The output signal of the human cardiac simulator has been recorded and compared with the ECGSIM output signal. All three standard recording methods are taken to produce $12 \mathrm{ECG}$ patterns. The signals pattern has met the specification of the ECGSIM signal. The human cardiac simulator also able to generate an irregular signal as an abnormal rhythm signal.

\section{REFERENCES}

[1] D. Sangita, G. Rajarshi and M. Mudhuchhanda, "Development of an Analog ECG Simulator Using Standalone Embedded System", International Kournal of Electrical, Electronics and Computer Engineering 1(2), pp. 83-87, 2012.

[2] Berbari, E. J., "Principles of Electrocardiography", Second Edition : "The Biomedical Engineering Handbook", Joseph D Bronzino, Indiana University/Purdue University, Indianapolis, pp.13.1-13.11, 2000.

[3] J. Zhang, "The Design of ECG Signal Generator using PIC24F," International Conference on Advances in Engineering 24, pp. 523-527, 2011.

[4] I. Valais, G. Koulouras, G. Fountos, C. Michail, D. Kandris and S. Athinaios, "Design and COnstruction of a Prototype ECG Simulator," eJournal of Science \& Technology (e-JST) 3(9), pp. 11-18, 2014.

[5] V. Oosterom, T. Oostendrop, P. Dam, "ECGSIM", Tersedia: Http://www.ECGSIM.org /index.php

[6] R. Firmansyah, B. Guruh and Sumber, "Monitoring Heart Rate And Temperature Based On The Internet Of Things ", j.electron.electromedical.eng.med.inform, vol. 1, no. 2, pp. 1-7, Oct. 2019.

[7] R. Alvionita, R. Ais, I. Wisana and Triwiyanto "Design of Cardiac Monitor for Multi Parameters", International Seminar on Application for Technology of Information and Communication (iSemantic), Semarang, Indonesia, pp. 423-428, 2019. 